\title{
Experimental Evidence of Delocalized States in Random Dimer Superlattices
}

\author{
V. Bellani, ${ }^{1}$ E. Diez,${ }^{2}$ R. Hey,${ }^{3}$ L. Toni,${ }^{4}$ L. Tarricone,${ }^{4}$ G. B. Parravicini,${ }^{1}$ \\ F. Domínguez-Adame, ${ }^{5}$ and R. Gómez-Alcalá ${ }^{6}$ \\ ${ }^{1}$ INFM-Dipartimento di Fisica "A. Volta," Universitá di Pavia, I-27100 Pavia, Italy \\ ${ }^{2}$ GISC, Departamento de Matemáticas, Universidad Carlos III de Madrid, E-28911 Leganés, Spain \\ ${ }^{3}$ Paul Drude Institut für Festkörperelektronik, Hausvogteiplatz 5-7, D-10117 Berlin, Germany \\ ${ }^{4}$ INFM-Dipartimento di Fisica, Universitá di Parma, I-43100 Parma, Italy \\ ${ }^{5}$ GISC, Departamento de Física de Materiales, Universidad Complutense, E-20840 Madrid, Spain \\ ${ }^{6}$ Departamento de Tecnologías de las Comunicaciones, Universidad de Vigo, E-36200 Vigo, Spain
}

(Received 21 July 1998; revised manuscript received 17 November 1998)

We study the electronic properties of GaAs-AlGaAs superlattices with intentional correlated disorder by means of photoluminescence and vertical dc resistance. The results are compared to those obtained in ordered and uncorrelated disordered superlattices. We report the first experimental evidence that spatial correlations inhibit localization of states in disordered low-dimensional systems, as our previous theoretical calculations suggested, in contrast to the earlier belief that all eigenstates are localized. [S0031-9007(99)08627-5]

PACS numbers: 73.20.Dx, 73.20.Jc, 78.55.-m, 78.66.-w

In recent years, a number of tight-binding [1-3] and continuous [4] models of disordered one-dimensional (1D) systems have predicted the existence of sets of extended states, in contrast to the earlier belief that all the eigenstates are localized in 1D disordered systems. These systems are characterized by the key ingredient that structural disorder is short-range correlated. Because of the lack of experimental confirmations, there are still some controversies as to the relevance of these results and their implications on physical properties. In this context, some authors have proposed finding physically realizable systems that allow for a clear cut validation of the above-mentioned purely theoretical prediction [5-8]. Given that semiconductor superlattices (SL's) have been already used successfully to observe electron localization due to disorder [9-14], these authors have suggested SL's as ideal candidates for controllable experiments on localization or delocalization and related electronic properties [5-8].

To the best of our knowledge, up to now there is no experimental verification of this theoretical prediction owing to the difficulty in building nanoscale materials with intentional and short-range correlated disorder. However, the confirmation of this phenomenon is important both from the fundamental point of view and for the possibility to develop new devices based on these peculiar properties. In this work we present an experimental verification of this phenomenon in semiconductor nanoscale materials, taking advantage of the molecular beam epitaxy growth technique, which allows the fabrication of semiconductor nanostructures with monolayer controlled perfection.

We grew several GaAs- $\mathrm{Al}_{0.35} \mathrm{Ga}_{0.65} \mathrm{As}$ SL's and we studied their electronic properties by photoluminescence (PL) at low temperature and dc vertical transport in the dark. Indeed PL has been proven to be a good technique to study the electronic properties of disordered SL's [9-11], giving transition energies comparable with theoretical calculations of the electronic levels. The electronic states were calculated using a Kronig-Penney model that has been shown to hold in this range of well and barrier thicknesses, with precise results [15]. This allows the analysis of the experimental transition energies for PL and the ascertainment of the localization and delocalization properties of the SL's. The details of the calculations and a schematic view of the conductionband profiles of the three SL's can be found in Ref. [7].

The samples are three undoped SL's grown by molecular beam epitaxy. All the SL's have 200 periods and $\mathrm{Al}_{0.35} \mathrm{Ga}_{0.65} \mathrm{As}$ barriers $3.2 \mathrm{~nm}$ thick. In the ordered SL all the 200 wells are identical with thickness $3.2 \mathrm{~nm}$ (hereafter referred to as $A$ wells). In the random SL, $58 A$ wells are replaced by wells of thickness $2.6 \mathrm{~nm}$ (hereafter referred to as $B$ wells) and this replacement is done randomly. The so-called random dimer SL is identical to the random SL with the additional constraint that the $B$ wells appear only in pairs [7]. In the latter sample the disorder exhibits the desired short-range spatial correlations. In each sample, the SL is clad on each side by $100 \mathrm{~nm}$ of $n-\mathrm{Al}_{0.3} \mathrm{Ga}_{0.7} \mathrm{As}$, Si doped to $4 \times 10^{18} \mathrm{~cm}^{-3}$, with a $50 \mathrm{~nm} n$-GaAs buffer layer (doped to $4 \times 10^{18} \mathrm{~cm}^{-3}$ ) on the substrate and a $3 \mathrm{~nm}$ $n$-GaAs cap layer (doped to $6 \times 10^{18} \mathrm{~cm}^{-3}$ ).

We measured x-ray diffraction spectra of the SL's with a double-crystal diffractometer, in order to check their structural parameters. The diffraction curve at (004) symmetric reflections for the two disordered samples shows satellite peaks of order \pm 1 lying close to $\pm 0.8^{\circ}$ with respect to the GaAs peak. These satellite peaks are located at identical positions for the two disordered SL's, showing that the random SL's have identical periods. Therefore, the dimer constraint intentionally introduced 
during sample growth is the only difference between random and random dimer samples.

The PL spectra were taken in the 11-300 K temperature range with a closed cycle cryostat and were excited with $514.5 \mathrm{~nm}$ light from an $\mathrm{Ar}^{+}$-ion laser (with an excitation intensity of approximately $0.5 \mathrm{~W} / \mathrm{cm}^{2}$ ). Photoluminescence was dispersed by a $0.46 \mathrm{~m}$ Jobin Yvon monochromator and detected by a cooled photomultiplier using a standard lock-in technique.

Figure 1 shows the PL spectra of the three SL's at $11 \mathrm{~K}$. We observed the fact that the energy of the nearband edge peaks depends on the sample, but the energy shift between them is almost independent of temperature on a wide range, as shown in Fig. 2. The PL peak for the ordered SL, which lies at $1.688 \mathrm{eV}$, is due to a recombination between electrons in the conduction band and heavy holes in the valence band [16]. We calculated the miniband structure of this SL with the Kronig-Penney model, using $\Gamma$ effective masses (in units of free electron mass) $m_{e}^{*}=0.067$ for GaAs and $m_{e}^{*}=$ 0.096 for $\mathrm{Al}_{0.35} \mathrm{Ga}_{0.65} \mathrm{As}$. The expected miniband in the conduction band lies in the range between 1.68 and $1.76 \mathrm{eV}$, measured from the (very narrow) heavy hole miniband. This calculation is in good agreement with the experimental PL spectrum, with the calculated lower energy of the miniband being very close to the energy at which PL intensity rises up. Figure 3(a) shows an energy schema of the radiative transitions in the ordered SL.

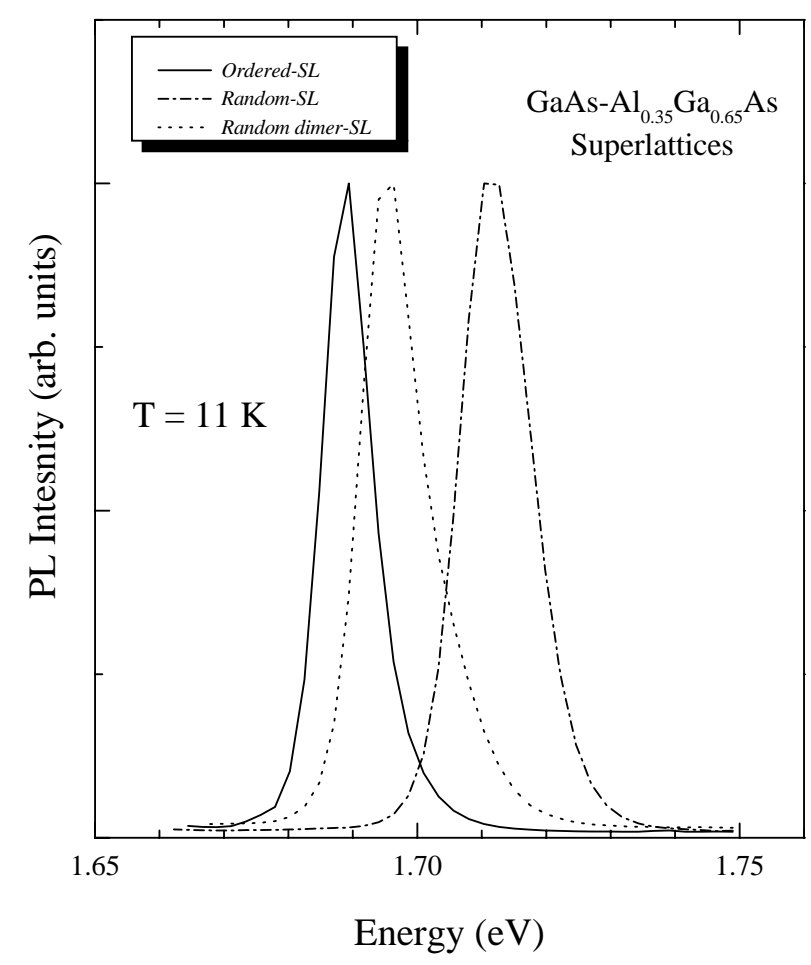

FIG. 1. PL spectra of the ordered, random, and random dimer SL at $11 \mathrm{~K}$.
Let us now analyze the spectrum obtained for the random SL. The PL peak of this sample shifts towards higher energies compared with the other two samples. In the random SL the intentional disorder introduced by the random distribution of wells $B(2.6 \mathrm{~nm})$ localizes the electronic states [7]. The calculated energy for the transition between electron and holes in this case is $1.72 \mathrm{eV}$ assuming that the exciton binding energy is the same in the three SL's. This value is again in excellent agreement with the PL peak, as can be seen in Fig. 1. Figure 3(b) shows a schematic diagram of the radiative transitions between localized states in the random SL.

The PL peak of the random dimer SL is at $1.696 \mathrm{eV}$ and, as can be clearly seen in Fig. 1, is redshifted with respect to the PL peak for the random SL. As has been shown by Fujiwara [15], the redshift of the PL peak in semiconductor SL's is due to the formation of a miniband with a tunnel process for carriers between the GaAs wells. This result strongly supports previous theoretical predictions of the occurrence of a band of extended states in random dimer SL's [7]. We calculated the transmission coefficient for the random dimer SL according to Ref. [7] and found that the energy difference between the onset for electron delocalization (that is, the energy at which transmission suddenly rises, as can be seen in Fig. 4) and the heavy hole miniband is around $1.70 \mathrm{eV}$, in good agreement with the experimental PL peak energy. Figure 3(c) presents a schematic diagram of the radiative transitions in the random dimer SL.

Additionally, the PL linewidth gives support to these findings. The PL full width at half maximun of the ordered SL is $9.1 \mathrm{meV}$, increasing to 13.2 and $12 \mathrm{meV}$ in the random SL and random dimer SL, respectively, indicating that these last two samples reflect their intentional disorder in the optical spectra.

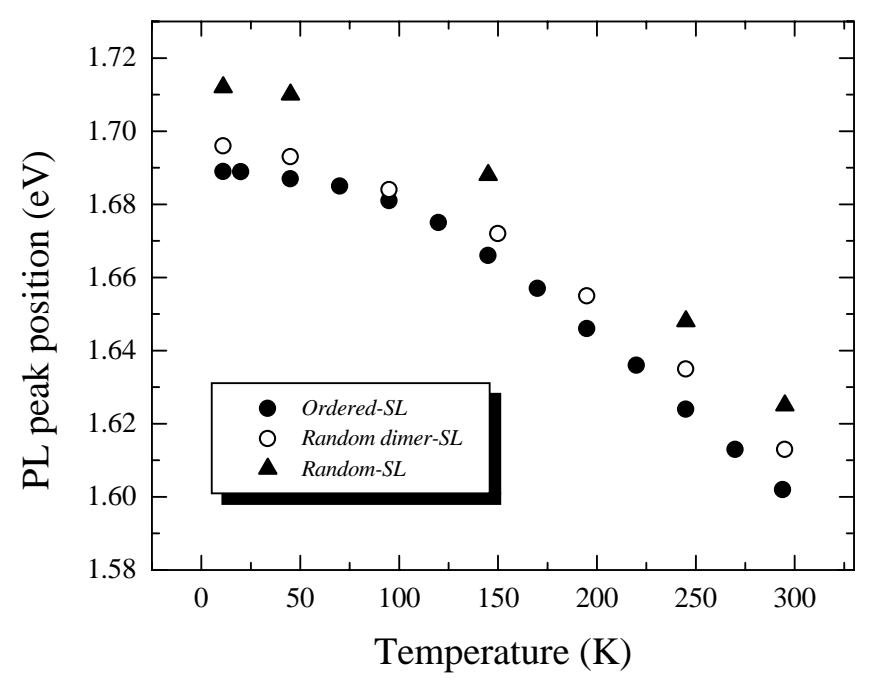

FIG. 2. Temperature dependence of the PL peak position of the ordered, random, and random dimer SL. 


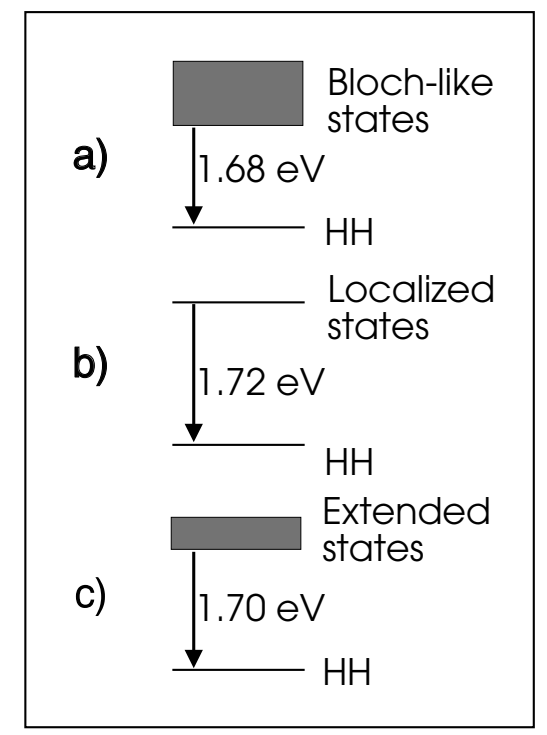

FIG. 3. Schematic diagram of radiative transitions in (a) ordered SL, (b) random SL, and (c) random dimer SL at $11 \mathrm{~K}$.

To confirm the above interpretation of the PL spectra we have performed additional measurements of the resistance at low temperatures [17]. The results for the temperature dependence of the resistance are shown in Fig. 5. The resistance of the random dimer SL is very similar to the resistance of the ordered SL for any temperature below $40 \mathrm{~K}$, and the small differences are due to the different miniband width between both SL's (see Fig. 3). On the other hand, the random SL shows a much higher resistance in this range of temperatures. This is completely consistent with the above interpretation of the PL spectra and it is clear evidence of the presence of extended states in the random dimer SL showing transport properties very similar to an ordered SL. Moreover, the resistance of the random SL still depends on temperature below $30 \mathrm{~K}$, while the resistance of the two other samples reaches a plateau. For low temperatures, transport properties in the presence of true extended states should be independent of temperature [17] and, as can be seen in Fig. 5, this behavior is observed only in the random dimer SL and in the ordered SL, which is additional evidence of the presence of extended states in these samples.

In summary, we have observed the fact that the introduction of short-range correlations in a disordered semiconductor SL inhibits localization and gives rise to extended states, as expected theoretically [7]. The positions of the electronic levels were calculated with the Kronig-Penney model and the calculations show that the ordered SL and the random dimer SL exhibit extended electronic states. According to theoretical studies, these extended states in random dimer SL's are not Bloch-like, as occurs in ordered SL's.

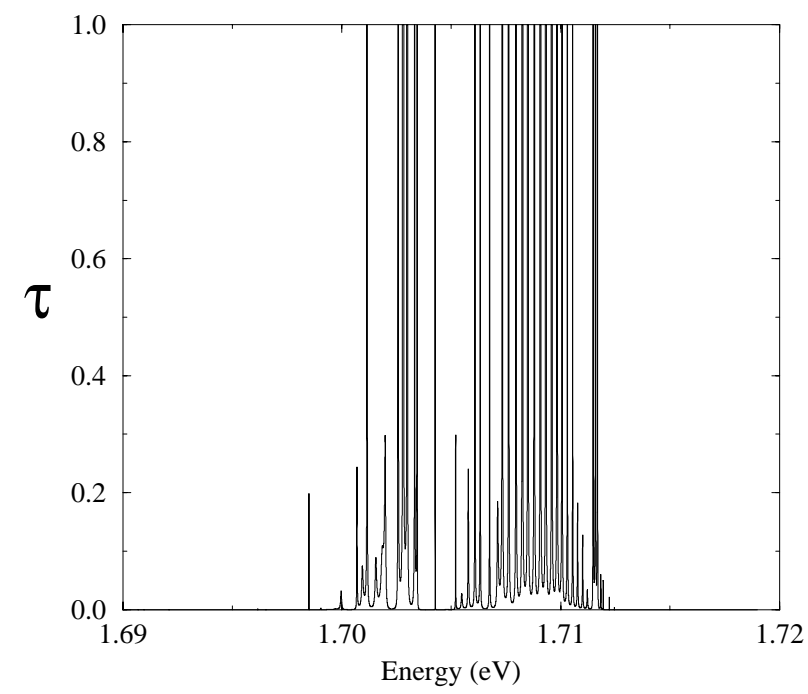

FIG. 4. Calculated transmission coefficient as a function of the electron energy in the random dimer SL. Energy is measured from the heavy hole miniband.

The PL of the random dimer SL is redshifted with respect to the PL of the random SL, indicating the formation of delocalized extended states. The experimental PL energies are in very good agreement with the calculated electronic states. The temperature dependence of the resistance of the random dimer SL is very similar to that of the ordered SL. Both SL's show no temperature dependence below $30 \mathrm{~K}$ as should be expected for transport in the presence of extended states. On the contrary, the resistance of the random SL is much higher for any temperature and shows temperature dependence as would be expected for localized states. To conclude, we have experimentally validated the existence of extended states in low-dimensional random systems with short-range correlations, where Anderson localization is inhibited.

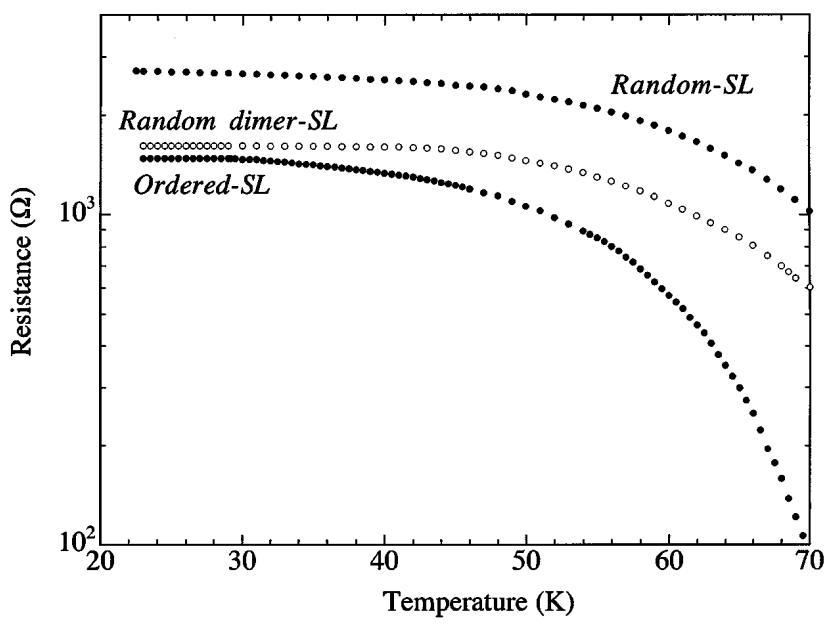

FIG. 5. Temperature dependence of the dc resistance of the ordered, random, and random dimer SL. 
Work in Italy has been supported by the INFM Network "Fisica e Tecnologia dei Semiconduttori III-V" and in Madrid by the CAM under Project No. 07N/0034/1998. E. D. and F. D.-A. thank A. Sánchez for collaboration on these topics during these years.

[1] J. C. Flores, J. Phys. Condens. Matter 1, 8479 (1989).

[2] D. H. Dunlap, H.-L. Wu, and P. Phillips, Phys. Rev. Lett. 65, 88 (1990).

[3] P. Phillips and H.-L. Wu, Science 252, 1805 (1991).

[4] A. Sánchez, E. Maciá, and F. Domínguez-Adame, Phys. Rev. B 49, 147 (1994).

[5] E. Diez, A. Sánchez, and F. Domínguez-Adame, Phys Rev. B 50, 14359 (1994).

[6] F. Domínguez-Adame, A. Sánchez, and E. Diez, Phys. Rev. B 50, 17736 (1994).

[7] E. Diez, A. Sánchez, and F. Domínguez-Adame, IEEE J. Quantum Electron. 31, 1919 (1995).
[8] E. Diez, A. Sánchez, F. Domínguez-Adame, and G.P. Berman, Phys. Rev. B 54, 14550 (1996).

[9] A. Chomette, B. Deveaud, A. Regreny, and G. Bastard, Phys. Rev. Lett. 57, 1464 (1986).

[10] L. Pavesi, E. Tuncel, B. Zimmermann, and F. K. Reinhart, Phys. Rev. B 39, 7788 (1989).

[11] Y.A. Zhang, J. A. Strozier, Jr., and A. Ignatiev, Phys. Rev. B 53, 7426 (1996).

[12] G. F. Lorusso, V. Capozzi, and J. L. Staehli, Semicond. Sci. Technol. 11, 308 (1996).

[13] K. A. Mäder, L.-W. Wang, and A. Zunger, Phys. Rev. Lett. 74, 2555 (1995).

[14] K. A. Mäder, L.-W. Wang, and A. Zunger, J. Appl. Phys. 78, 6639 (1995).

[15] K. Fujiwara, in Semiconductor Superlattices, edited by H. T. Grahn (World Scientific, Singapore, 1995).

[16] Exciton binding energies are much smaller than the observed peak shifts.

[17] G. Richter, W. Stolz, P. Thomas, S. W. Koch, K. Maschke, and I.P. Zvyagin, Superlattices Microstruct. 22, 475 (1997). 